selbst diesem „Stamm“ in gewisser Weise an. Zudem stellt sich die Frage, ob man eine andere Sprache für die Beschreibung der Soziologie finden kann als die Sprache der Soziologie selbst.

\title{
2.3 THEORETISCHE KONSEQUENZEN
}

Aus diesen Ausführungen ergibt sich eine Reihe von Konsequenzen für die weitere Arbeit. Zunächst werden hier die theoretischen Implikationen dargestellt. Was ergibt sich für die weitere Untersuchung von Grenzen zwischen der Soziologie und ihrem Untersuchungsgegenstand? Welche theoretischen Schlussfolgerungen lassen sich aus den Kapiteln 2.1 und 2.2 ziehen? Wie aus den vorhergehenden Abschnitten deutlich geworden ist, haben beide Ansätze, die Systemtheorie wie auch die STS, spezifische Vorstellungen von Grenzen und Grenzbestimmungen zwischen Wissenschaft und Nicht-Wissenschaft. Zudem hat sich gezeigt, dass jeder Ansatz für sich auch gewisse Limitierungen aufweist. Deshalb werden in diesem Kapitel die beiden Ansätze in ihren Konvergenzen und Divergenzen reflektiert. Außerdem wird in Hinblick auf die Forschungsfrage der theoretische Ansatz spezifiziert, mit dem weitergearbeitet werden soll. In einem nächsten Schritt ergeben sich auch methodologische Konsequenzen: Was sagt das theoretische Konzept für die weitere empirische Bearbeitung der Fragestellung aus?

\subsubsection{Konvergenzen und Divergenzen zwischen Selbstbeschreibungen und Grenzarbeit}

Um sich nicht von vornherein auf eine epistemologische Perspektive festzulegen, wurden zwei unterschiedliche Theoriestränge eingebracht. Denn so geeignet das Konzept der Selbstbeschreibungen auf den ersten Blick erscheinen mag, so wichtig ist es dennoch, die theoretischen und epistemologischen Konsequenzen zu reflektieren, die mit der Übernahme einer solchen Perspektive einhergehen. Deshalb wurde eine zweite Sichtweise hinzugezogen, die der Systemtheorie zunächst wenig verwandt erscheint: die der Science and Technology Studies, vornehmlich das Konzept der boundary work von Gieryn (1983; 1995; 1999). Der Vergleich der beiden Zugänge erlaubt eine kritische Reflexion derselben. Wir haben bereits im vorhergehenden Zwischenfazit einiges über die epistemologischen Grundlagen der Systemtheorie im Vergleich zu den STS erfahren. Im weiteren Schritt sollen Konvergenzen und Divergenzen zwischen Systemtheorie und den STS, insbesondere dem Konzept der boundary work, erläutert werden. 
Lassen wir zunächst die Divergenzen außer Acht und blicken auf die Konvergenzen. Für die Systemtheorie wie auch für das Konzept der boundary work sind Grenzen essenziell. Bei beiden werden Grenzen über Sprache erzeugt, in der Systemtheorie in Form von Semantiken, in der boundary work über Rhetoriken. Sprache ist für beide sinnevozierend. Sie adressiert dahinterliegende Sinnstrukturen und gewährleistet damit den Anschluss an weitere (System-)Operationen.

Dennoch unterscheiden sich Selbstbeschreibungen und boundary work in ihrer epistemischen Perspektive, in der Art und Weise, wie sie Grenzziehungen verstehen, in ihrem Verständnis von Semantik und Rhetorik und darin, inwiefern sie die Soziologie/Sozialwissenschaften als einen spezifischen Fall begreifen (siehe Tabelle 3). 
Tabelle 3: Unterschiede zwischen Selbstbeschreibungen und Boundary Work

\begin{tabular}{|c|c|c|}
\hline & Selbstbeschreibungen & Boundary Work \\
\hline $\begin{array}{l}\text { Epistemische Per- } \\
\text { spektive }\end{array}$ & $\begin{array}{l}\text { - } \text { Blick von innen } \\
\text { - In sich geschlossen } \\
\text { operierende Systeme } \\
\text { - System im Mittel- } \\
\text { punkt } \\
\text { - Anwendung der } \\
\text { Systemtheorie auf } \\
\text { sich selbst } \\
\text { - Beobachtung zwei- } \\
\text { ter Ordnung (blinder } \\
\text { Fleck der Beobach- } \\
\text { tenden) }\end{array}$ & 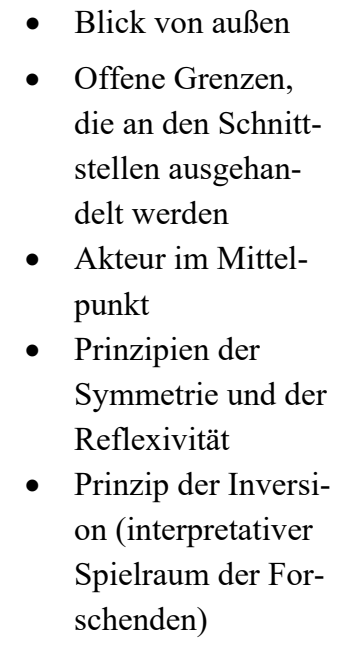 \\
\hline Grenzkonstruktion & $\begin{array}{l}\text { - Grenzen über Sys- } \\
\text { tem-Umwelt- } \\
\text { Differenz } \\
\text { - Grenzen stabil }\end{array}$ & $\begin{array}{l}\text { - Grenzkonstruktion } \\
\text { über Demarkation } \\
\text { von Wissenschaft } \\
\text { und Nicht- } \\
\text { Wissenschaft } \\
\text { - Grenzen volatil }\end{array}$ \\
\hline Semantik/Rhetorik & $\begin{array}{ll}\text { - } & \text { Selbstaffirmativ } \\
\text { - } & \text { Sinnerzeugend zum } \\
& \text { Systemerhalt } \\
\text { - } & \text { Selbstreferenziell } \\
\text { - } & \text { Emergent }\end{array}$ & $\begin{array}{ll}\text { - } & \text { Kontrovers } \\
\text { - } & \text { Strategisch einge- } \\
\text { setzt (,credibility } \\
\text { contests) } \\
\text { - } \quad \text { Fremdreferenziell } \\
\text { - ,in the making“ }\end{array}$ \\
\hline $\begin{array}{l}\text { Soziologie als be- } \\
\text { sonderer Fall? }\end{array}$ & $\begin{array}{l}\text { - Besonderes Verhält- } \\
\text { nis zur Gesellschaft: } \\
\text { System im System } \\
\text { - Selbstbeschreibung } \\
\text { und Fremdbeschrei- } \\
\text { bung }\end{array}$ & $\begin{array}{l}\text { Disziplin neben an- } \\
\text { deren Disziplinen } \\
\text { - Besonderer Legiti- } \\
\text { mierungsbedarf } \\
\text { aufgrund des Ver- } \\
\text { dachts der „Pseu- } \\
\text { dowissenschaft“ }\end{array}$ \\
\hline
\end{tabular}




\subsubsection{Rolle von Rhetorik und Semantik}

In Kapitel 2.1 haben wir eine Reihe unterschiedlicher Semantiken erlebt, die im historischen Kontext als spezifische Selbstbeschreibungen der Soziologie gelten. Sie wurden im Luhmann'schen Sinn als Beschreibungen fixiert, als bewahrenswert anerkannt und für die Wiederholung bereitgehalten. Wir haben einige solcher Semantiken kennengelernt, etwa die der „Sozialtechnologie“ und „Aufklärung" als Selbstbeschreibung der Soziologie, aber auch die von „Objektivität“ und „Normativität“ sowie „Theorie“ und „Praxis“ als Selbst- wie Fremdbeschreibungen, die als Dichotomie in einem dialektischen Verhältnis zueinander stehen. Als diverse Semantiken eines Diskurses stellen sie einen „Vorrat an Sinnverarbeitungsregeln“ bereit. In Kapitel 2.1 haben wir ebenso gesehen, dass die Selbstbeschreibungen an bereits bestehende Kommunikation anknüpfen. Im Fall des Werturteilsstreits sind die Postulate der Wertfreiheit und des Wertbezugs von Forschung auf die vorhergehenden wissenschaftstheoretischen Debatten und historischen Ereignisse zu beziehen. Schon allein die Namensgebung der Soziologie ist historisch kontingent: Die von Auguste Comte entwickelte Bezeichnung ,sociology“ entstand unter den Vorzeichen des Positivismus und verortete die Soziologie (im Sinn einer sozialen Physik) als strenge Wissenschaft, die ähnlich wie die Physik übergeordnete Gesetze ableitet, wie die Gesellschaft funktioniert (Giddens et al., 2009b). Mit einer Selbstbeschreibung des Subsystems „sociology“ als Zusammensetzung der Termini „socius“, „sociālis“ (lat: gemeinsam, die Gesellschaft betreffend) und „logos“ (griech: wissenschaftliche Disziplin, Fachwissenschaft) (DWDS, o.J.; Diaz-Bone, 2010) wurde die Soziologie semantisch also bereits den Wissenschaften zugeordnet.

Die vorhergehenden Ausführungen haben klargemacht, dass die analytische Perspektive der Systemtheorie deutliche Nachteile hat: Zunächst kann sie Semantiken nur auf Ebene des Systems bestimmen. Sie sind systememergent. Semantiken in Form von Selbstbeschreibungen sind als Operationen des Systems zu verstehen. Aus dieser Perspektive lässt sich die semantische Ebene der historischen Debatten um die Rolle der Soziologie in der Gesellschaft durchaus nachvollziehen. Wie wir bereits gehört haben, postulierte Luhmann, dass Selbstbeschreibungen in modernen Gesellschaften in Schriftform vorliegen müssen. Wenn sie in Form des generalisierten Kommunikationsmediums der Publikation vorliegen, bleiben sie als Reflexionstheorien auf der Ebene des Wissenschaftssystems. Nicht nachvollziehbar ist in diesem Zusammenhang der gezielte Einsatz dieser Semantiken, um bestimmte Ziele zu erreichen, sowie die Frage des Einflusses und der Deutungshoheit und damit auch ihr Einfluss auf der Mikroebene. 
Diese Lücke adressiert letztlich das Konzept der boundary work, indem es untersucht, wie diverse Rhetoriken eingesetzt werden. Grenzarbeit geschieht also nicht nur selbstreferenziell im System, sondern findet ihre Anwendung als ein „rhetorical style“. Die Rhetorik dieser „ideologischen Selbstbeschreibungen“, wie Gieryn sie nennt, bewegt sich jedoch nicht im sinnentleerten Raum, sondern knüpft gezielt an bestimmte bereits existierende Sinnzuschreibungen an, etwa an die einer „moral economy“ der Wissenschaft. Die damit verbundenen historischen „Sedimentierungen“ bezeichnen einen kartografischen Bedeutungsraum, in dem die Grenzarbeit stattfindet. Semantiken sind dem Konzept der boundary work also nicht gänzlich fremd, sie sind nur anders kontextuiert: Sie zeichnen sich dadurch aus, dass sie multiple Bedeutungen haben und unterschiedliche Rhetoriken flexibel einsetzen. Beispielhaft steht hierfür Calverts Konzept des „tailoring“ (Calvert, 2006, S. 208).

Rhetorik funktioniert im Gegensatz zu Semantiken nur mit ,receptive audiences“ (Fuller, 1991, S. 308), ist also angewiesen auf ein responsives Publikum. Hier wird der fremdreferenzielle Charakter der Rhetorik deutlich: Sie richtet sich immer an ein Äußeres und reagiert, je nach Bedarf, auch auf dieses Äußere. So werden Selbstbeschreibungen nicht zu selbstaffirmativen sinnerhaltenden Systemoperationen, sondern stellen ,ideological self-descriptions“ dar (Gieryn, 1983, S. 783). Selbstbeschreibungen sind also auch dem Konzept der Grenzarbeit bekannt. Aus Sicht der Grenzarbeit jedoch macht es einen Unterschied, wer die Selbstbeschreibung anfertigt und zu welchem Zweck. Nach Calvert können diese wiederum selbstreferenziell werden in dem Sinn, dass sie als Selbstbeschreibungen auch identitätsstiftenden Charakter haben. Grenzarbeit also nicht nur über die Systemfrage zu adressieren, sondern auch auf der Mikroebene, hat deshalb entscheidende analytische Vorteile: Es erlaubt einen Blick darauf, auf welche Art und Weise bestimmte Semantiken über Rhetoriken eingesetzt werden.

Mithilfe des boundary work-Konzepts ist es möglich, einer weiteren Schwäche der Systemtheorie zu begegnen. Luhmann geht davon aus, dass Semantiken dem System nachgelagert sind. Jedoch ist es gerade mithilfe des unterschiedlichen Einsatzes bestimmter Rhetoriken möglich, verändernde Sinnangebote genauer zu betrachten und die Dynamik derselben zu verfolgen. Im Gegensatz zum systemtheoretischen Verständnis von Selbstbeschreibungen zeigt boundary work, wie bspw. die Soziologie ständig Differenz zu Nicht-Soziologie herstellt. So wirkte der Werturteilsstreit sinnstiftend auf die Soziologie und hat weitere Systemoperationen wie auch Problemwahrnehmungen im System geprägt (beispielsweise den Positivismusstreit, der auch zweiter Werturteilsstreit genannt wird). Der Werturteilsstreit, der nicht zwangsläufig auf der „Ursprungssemantik“ 
beruht, kann aber auch als rhetorische Ressource zur Begründung einer bestimmten Grenzziehung zwischen Soziologie und Gesellschaft eingesetzt werden. Akteure können also eine bestimmte Semantik in entscheidendem Maße verändern, die sich möglicherweise - wenn überhaupt - im Nachhinein erst in Schriftform niederschlägt, und damit wieder Operationen im System bzw. das gesamte System selbst beeinflussen, was Stäheli die „lineare Nachträglichkeit“ der Semantik nennt (vgl. Kapitel 2.3.3.1.4). Hier sind wir bereits sehr nah an einer Grundannahme, wie sie Bourdieu in seiner Theorie der Praxis oder auch Giddens in seiner Strukturationstheorie geäußert hat: Die sogenannte Mikro- und Makroebene beeinflussen einander, objektive Strukturen und subjektive Orientierungen sind nicht voneinander zu trennen (Bourdieu, 2015[1979]; Bourdieu, 2016[1982]; Giddens, 1984).

Dennoch muss man fragen, ob das boundary work-Konzept nicht die Rolle von sinnstiftender Semantik unterbewertet. Denn obwohl Gieryn implizit auf die Rolle von sinnstiftenden Ideologien bei der Grenzarbeit hinweist, ist für ihn alle Grenzarbeit grundlegend strategisch angelegt. Werden Rhetoriken aber wirklich so bewusst genutzt? Oder hat die Nutzung einer bestimmten Semantik nicht doch auch mit einer Form der Selbstbeschreibung zu tun, die mehr sinn- und identitätsstiftend als rein „nutzenorientiert“ ist? Dem wird hier empirisch auf den Grund gegangen.

Mit Blick auf die Rolle von Rhetorik und Semantik in Systemtheorie und boundary work lässt sich aber zunächst festhalten, dass das Zusammenführen von Systemtheorie (Semantik) und Grenzarbeit (Rhetorik) zu einem theoretischen und analytischen Mehrwert führt.

\subsubsection{Die doppelte Konstitution der Soziologie}

Die Analyse der Soziologie im Kontext einer soziologischen Arbeit weist einige Besonderheiten auf. In den soziologischen Debatten wurde die spezielle Stellung herausgehoben, die die Soziologie in Hinblick auf ihren Untersuchungsgegenstand hat (siehe u.a. Bourdieu et al., 1991; Beck, 1974; Beck et al., 1989a; Gouldner, 1968; 1970; Giddens, 1976; 1984; Schelsky, 1959; 1975; 1981; Luhmann, 1993; Kieserling, 2004). Diese Debatten stellen aus systemtheoretischer Sicht Reflexionstheorien dar, die Bedingungen soziologischer Wissensproduktion diskutieren. Diese Reflexionstheorien beinhalten einerseits methodologische Überlegungen, wie diese Art von ,unreiner" Soziologie zu vermeiden ist. Andererseits reflektieren theoretische Überlegungen das Verhältnis zwischen Soziologie und ihrem Untersuchungsgegenstand: Sie ist in der einen oder anderen Weise mit ihrem Untersuchungsgegenstand verbunden. Ausgangspunkt dieser Reflexi- 
onstheorien ist die Annahme, dass soziologisches Wissen wie eine Art ,parasitäres“ Wissen auf anderen Wissensformen aufsitzt, von denen es sich dann wiederum distanzieren muss. Ein Großteil der selbstreflexiven Unterfangen der Soziologie beschäftigt sich also damit, professionalisiertes soziologisches Wissen ontologisch von anderen Wissensformen zu differenzieren und sich damit sein eigenes Terrain zu sichern und zu legitimieren. Geschieht dies nicht, werden einige Gefahren deutlich: Bourdieu et al. sprechen von der Gefahr der „Spontansoziologie“" (Bourdieu et al., 1991, S. 15). Gemeint ist eine Soziologie, die nicht theoretisch und methodologisch fundiert ist und Alltagsmeinungen vertritt:

„Die Trennung zwischen alltäglicher Wahrnehmung und Wissenschaft, die sich etwa für den Physiker im entschiedenen Gegensatz von Alltagsleben und Laboratorium niederschlägt, fällt ihm [dem Soziologen] umso schwerer, als er im theoretischen Erbe, auf das er zurückgreifen kann, Hilfsmittel zur radikalen Zurückweisung der Alltagssprache und ihrer Begriffe nicht findet.“ (Bourdieu et al., 1991, S. 15)

Die Systemtheorie hat eine andere Antwort auf diese Frage. Die Soziologie kann sich nur innerhalb der Gesellschaft beschreiben. Sie kann nicht außerhalb der Gesellschaft vorkommen. Und: „Will man genauer wissen, wie sie in der Gesellschaft vorkommt, lautet die Antwort: als Wissenschaft. Die Soziologie hat keine andere Arbeitsgrundlage.“ (Luhmann, 1993, S. 252) Nichtsdestoweniger zeigt Luhmann anhand zweier Grundausrichtungen der Soziologie, dass sie sich entweder am Wissenschaftssystem oder am Gesellschaftssystem orientiert. Die erste Grundausrichtung beschreibt er als positivistisch („Was ist der Fall?"), die zweite als kritisch („Was ist dahinter?“). Im ersten Fall fragt die Soziologie nach latenten Strukturen und bezieht sich auf das System Wissenschaft. Im zweiten Fall ist sie am Gesellschaftssystem orientiert und möchte inkongruente Perspektiven darstellen, indem sie als kritische Soziologie verkündet, dass die Welt nicht so ist, wie sie erscheint (Luhmann, 1993). ${ }^{48}$ Diese zwei Perspektiven stellen für Luhmann ein Paradoxon dar, eine Unterteilung der Soziologie in Vorder- und Hinterbühne, in Latenz und Präsenz, die nach Luhmann weder richtig ist noch dem Fach die Möglichkeit zur Einheit eröffnet. Beide Perspektiven gehen davon aus, dass Forschende einen externen Standpunkt einnehmen, der Gesellschaftsbeschreibung realisierbar macht. Verbunden damit ist ein „Besserwissertum“ über Gesellschaft, das insbesondere in Zeiten der Postmoderne obsolet ist.

48 Interessant ist hier auch, dass Luhmann mit dieser Unterscheidung eine dominante Selbstbeschreibung innerhalb der Soziologie (Sozialtechnologie und Aufklärung) reproduziert, andere Möglichkeiten der Selbstbeschreibung jedoch nicht nennt. 
Laut Luhmann kann die Soziologie jedoch keine der Gesellschaft externe Position einnehmen: Da sie immer interne Beobachterin des Systems ist, da sie Teil des Objekts ist, das sie beschreibt, gelten die klassischen Subjekt-ObjektUnterscheidungen nicht. Die Soziologie hat also ein ontologisches Problem. Denn obwohl sie beobachtet, kann sie sich nicht als unabhängige Reflexionsinstanz begreifen. Versteht man, so Luhmann, Gesellschaft als ein operativ geschlossenes System, ${ }^{49}$ so könnte kein externer Beobachter vorausgesetzt werden. Soziologie als Wissenschaft jedoch kann deshalb lediglich zur internen Beobachtung des Systems beitragen. Für Luhmann wird folglich das Problem der Ontologie zum Zustand der Autologie: Aufgrund der eigenen Disposition zum Objekt, der Gesellschaft, in das die Soziologie ja eingeschlossen ist, wird sie „ständig zu ,autologischen' Schlüssen gezwungen - zu Schlüssen von ihrem Gegenstand auf sich selber" (Luhmann, 1993, S. 255). Diese autologischen Schlüsse sind nach Kieserling (2004) die Bezüge der Selbst- und Fremdbeschreibung der Soziologie. Die Soziologie hat immer einen doppelten Bezug, den auf sich und den auf die Gesellschaft, mit dem sie sich aber wieder (autologisch) selbst beschreibt.

Nun ist es jedoch so, dass diese Problematisierung im theoretischen Sinn wiederum ihre Schwierigkeiten in sich birgt. Denn übernehmen wir diese Sicht als dominante theoretische Perspektive dieser Arbeit, so kaufen wir uns zugleich auch alle weiteren autologischen wie ontologischen Selbstbeschreibungen ein, die diesen Reflexionen inhärent sind. Dieser Umstand wurde bereits in Kapitel 2.2.2 als Problem der Selbstreferenz markiert.

Die Science and Technology Studies gehen ganz anders mit der doppelten Bezugnahme um: Mit der Formulierung ihrer epistemologischen Grundposition umgehen sie dieses „Problem“ letztlich einfach. Nicht die Legitimität der Soziologie im wissenschaftlichen Diskurs im engeren Sinn ist für sie entscheidend. Sie gehen eher von einer grundlegenden Verzahnung zwischen Wissenschaft und Nicht-Wissenschaft aus - jegliche Form der Trennung diverser Werte, Prozesse und Vorgänge ist demnach als Demarkationsinstrument zu verstehen. Wissenschaft ist in der Gesellschaft, genauso wie die Gesellschaft in der Wissenschaft ist. Dennoch sehen die STS, dass die Sozialwissenschaften ein besonderes Legitimationsproblem gegenüber der Gesellschaft haben, weil es ihnen seit jeher deutlich schwerer fällt, eine Ideologie der Objektivität nachzuweisen als den Naturwissenschaften. Diese konnten eine Externalisierung ihres Untersuchungsgegenstandes wesentlich besser dokumentieren (Fuller, 1991; Shapin et al., 2011).

49 Zum Begriff operativ geschlossener Systeme und zur Differenz von offenen und geschlossenen Systemen siehe Luhmann, 1991b. 
Die Folge war, dass die Naturwissenschaften und die Konstruktion von Objektivität für die STS ein interessanterer Untersuchungsgegenstand wurden als die Sozialwissenschaften. Bloors Postulat der Reflexivität wurde demzufolge nicht eingelöst - wir haben bereits über vereinzelte entsprechende Versuche gesprochen. Diese sind fast noch häufiger in der Soziologie selbst zu finden. Dennoch hilft auch hier die Perspektive der STS weiter: Denn aus ihrem Blickwinkel ist die grundlegende Frage nicht, was eigentlich das Problem der Sozialwissenschaften ist, sondern wie dieses aus konstruktivistischer Sicht beschaffen ist. Diese Perspektive hilft also, einen Schritt zurückzutreten und soziologische Selbstbeschreibungen in einem anderen Licht zu betrachten. Doch auch die STS sind der Herausforderung ausgesetzt, wie sie eigentlich eine Wissenschaft beschreiben sollen, deren Teil sie selber sind, wie sie Grenzarbeit analysieren sollen, ohne selbst wieder Grenzarbeit zu betreiben, und wie sie wissenschaftliche Theoriebildung und Laborbeobachtungen machen wollen, ohne selbst wiederum denselben (sozialen) Logiken und Selektionsprozessen ausgesetzt zu sein, die sie kritisieren.

Also stehen wir auch hier von dem Problem der doppelten Konstitution: Man ist selbst Teil dessen, was man beschreibt. Die STS reagieren darauf mit der „Inversion“, also einem wissenschaftstheoretischen Versuch, die eigenen Vorannahmen zu kontrollieren: Wissenschaft konstruiert den Gegenstand, nicht der Gegenstand die Wissenschaft. Inwieweit dies jedoch praktisch umgesetzt werden kann, ist wiederum die Frage. Dennoch: Wir sehen, dass die STS zwar analytisch eine hilfreiche Perspektive auf die Analyse der Soziologie in der Gesellschaft und die doppelte Konstitution der Soziologie anbieten. Allerdings sind sie den gleichen epistemologischen Herausforderungen in der praktischen Umsetzung der eigenen Arbeit ausgesetzt wie die Soziologie ${ }^{50}$ selbst. Die doppelte Konstitution der Soziologie bezieht sich also nicht nur auf ihre Doppelstruktur in Hinblick auf ihren Untersuchungsgegenstand, sondern auch auf die Notwendigkeit einer doppelten Reflexivität in Hinblick auf die Bedingungen ihrer Beobachtung. Von diesem Dilemma ist natürlich auch diese Arbeit nicht ausgenommen, und sie wird es auch nicht lösen können. Umso wichtiger erscheint es in diesem $\mathrm{Zu}$ sammenhang, die methodologischen Konsequenzen zu reflektieren, die sich aus den Ausführungen des vorliegenden Kapitels 2.3 ergeben.

50 Es wird an dieser Stelle selbst Grenzarbeit zwischen der Soziologie und den Science and Technology Studies betrieben. Ein Großteil der Ansätze der STS speist sich aus soziologischen Theorien, insbesondere der Wissenssoziologie. Dennoch sind die STS als interdisziplinäres und disperses Feld zu verstehen, das wesentliche Anreize ebenso aus der Wissenschaftsgeschichte und der Wissenschaftsphilosophie bezieht. 